\title{
Finite-time extinction of the Kähler-Ricci flow
}

\author{
JIAN SONG
}

\begin{abstract}
We investigate the limiting behavior of the unnormalized Kähler-Ricci flow on a Kähler manifold with a polarized initial Kähler metric. We prove that the Kähler-Ricci flow becomes extinct in finite time if and only if the manifold has positive first Chern class and the initial Kähler class is proportional to the first Chern class of the manifold. This proves a conjecture of Tian for the smooth solutions of the Kähler-Ricci flow.
\end{abstract}

\section{Introduction}

The limiting behavior of the Kähler-Ricci flow is deeply related to the existence of canonical metrics and the minimal model program in algebraic geometry. Let $X$ be an $n$-dimensional compact Kähler manifold. We consider the following unnormalized Kähler-Ricci flow starting with a Kähler metric $\omega_{0}$.

$$
\left\{\begin{array}{l}
\frac{\partial}{\partial t} \omega=-\operatorname{Ric}(\omega), \\
\left.\omega\right|_{t=0}=\omega_{0} .
\end{array}\right.
$$

If $c_{1}(X)=0$, then the unnormalized Kähler-Ricci flow (1.1) converges to the unique Ricci-flat metric in [ $\left.\omega_{0}\right]$ [Cao, Y1]. If $c_{1}(X)<0$, then the normalized Kähler-Ricci flow

$$
\frac{\partial}{\partial t} \omega=-\operatorname{Ric}(\omega)-\omega
$$

converges to the unique hyperbolic Kähler-Einstein metric [Cao, Y1, A].

If $c_{1}(X)$ is nonpositive and not strictly negative, then there does not exist a smooth Kähler-Einstein metric on $X$. However, the normalized Kähler-Ricci flow (1.2) has long-time existence. If $X$ is a minimal model of general type, then the Kähler-Ricci flow (1.2) converges to the unique singular Kähler-Einstein metric as time tends to infinity [Ts, TZha]. In general, if the canonical bundle $K_{X}$ is semi-ample, the normalized Kähler-Ricci 
flow (1.2) can possibly collapse but it always converges to the unique generalized Kähler-Einstein metric on the canonical model of $X$ [SoT1, SoT2].

If $c_{1}(X)>0, X$ is called a Fano manifold in algebraic geometry. It is conjectured by Yau [Y2] that the existence of a Kähler-Einstein metric is equivalent to suitable stability in the sense of geometric invariant theory. The condition of $K$-stability is later proposed by Tian [T1] and has been refined by Donaldson [D]. The Kähler-Ricci flow provides an approach to the Yau-Tian-Donaldson conjecture and it has attracted considerable current interest. We refer the reader to an incomplete list of literatures [PS1, PS2, PSSW1, PSSW2, Sz, To, CW, CS] for some recent development. If one assumes the existence of a Kähler-Einstein metric, then according to the unpublished work of Perelman [P2] (see [TZhu]), the normalized KählerRicci flow $\frac{\partial}{\partial t} g=-\operatorname{Ric}(g)+g$ with the initial Kähler metric in $c_{1}(X)$ converges to a Kähler-Einstein metric (this is due to $[\mathrm{H}, \mathrm{Ch}]$ in the case of one complex dimension).

Let us first define finite-time extinction for the Ricci flow.

Definition 1.1. Let $M$ be a closed Riemannian manifold and $g(t)$ be a smooth solution of the Ricci flow for $t \in[0, T)$. The Ricci flow is said to become extinct at $t=T$ if $(M, g(t))$ converges to a point in the sense of Gromov-Hausdorff, or equivalently, the diameter of $g(t)$ tends to 0 as $t \rightarrow T$.

It is proved in [IK] that the diameter of $g(t)$ is uniformly bounded away from 0 if there exists an element of infinite order in $H_{1}(M, \mathbf{Z})$. However, many projective manifolds do not admit elements of infinite order in $H_{1}(M, \mathbf{Z})$. In particular, Fano manifolds are all simply connected. Indeed, it is proved by Perelman [P1, P2] (see [SeT]) that the Kähler-Ricci flow (1.1) on Fano manifolds becomes extinct in finite time if the initial Kähler class is proportional to the first Chern class of the manifold. Thus it is of our interest to completely understand the extinction conditions for the KählerRicci flow (1.1). In general, the Kähler-Ricci flow can be weakly defined on singular projective varieties with mild singularities and surgeries might be performed, as investigated in [SoT3]. Therefore the notion of extinction can be generalized to the Kähler-Ricci flow with surgery. The following conjecture is proposed by Tian in [T2].

Conjecture 1.1. The Kähler-Ricci flow (1.1) with surgery becomes extinct in finite time if and only if the initial projective manifold is birationally equivalent to a Fano manifold. 
It is shown in $[\mathrm{SW}]$ that the conjecture holds in the special case of Hirzebruch surfaces when the initial Kähler metric satisfies the Calabi symmetry.

We prove Conjecture 1.1 in the case of the usual Kähler-Ricci flow with smooth solutions.

Theorem 1.1. Let $X$ be an n-dimensional Kähler manifold. Then the Kähler-Ricci flow (1.1) with an initial Kähler metric in the class of $H^{2}(X, \mathbf{Z})$ becomes extinct in finite time if and only if $X$ is Fano and the initial Kähler class is proportional to $c_{1}(X)$.

More generally, we have the following general diameter estimates.

Theorem 1.2. Let $X$ be an n-dimensional Kähler manifold. Let $\omega_{0}$ be a Kähler metric on $X$ such that $\left[\omega_{0}\right] \in H^{2}(X, \mathbf{Z})$. Then the diameter is uniformly bounded away from 0 along the Kähler-Ricci flow (1.1) if one of the following conditions holds.

1) $K_{X}$ is semi-ample.

2) $K_{X}$ is not nef and $K_{X}^{-1}$ is not ample.

3) $K_{X}^{-1}$ is ample and $\left[\omega_{0}\right]$ is not proportional to $\left[K_{X}^{-1}\right]$.

In particular, if $K_{X}$ is semi-ample and the Kodaira dimension of $X$ is positive, the diameter tends to infinity of order $\sqrt{t}$ as $t \rightarrow \infty$ along the Kähler-Ricci flow (1.1).

It is known that the abundance conjecture holds for dimension three and so $K_{X}$ is semi-ample whenever it is nef. The following corollary is then an immediate consequence of Theorem 1.2.

Corollary 1.1. Let $X$ be a Kähler manifold of $\operatorname{dim} X \leq 3$. Then the KählerRicci flow (1.1) on $X$ with an initial Kähler metric in $H^{2}(X, \mathbf{Z})$ becomes extinct if and only if $X$ is Fano and the initial Kähler class is proportional to $c_{1}(X)$.

The above statement holds for $\operatorname{dim} X \geq 4$ if we assume the abundance conjecture.

We further propose the following conjecture as a natural attempt to generalize Theorem 1.1 and Corollary 1.1 by removing the condition for the initial Kähler class and the assumption of the abundance conjecture. 
Conjecture 1.2. Let $X$ be an $n$-dimensional Kähler manifold. Then the Kähler-Ricci flow on $X$ with an initial Kähler metric becomes extinct if and only if $X$ is Fano and the initial Kähler class is proportional to $c_{1}(X)$.

Remark 1.1 Theorem 1.1 also holds if the initial Kähler metric is in a multiple of a class in $H^{2}(X, \mathbf{Z})$. This can be easily proved by suitable scaling of the unnormalized flow (1.1). It is pointed out by V. Tosatti to the author that Theorem 1.1 should also be true if the initial Kähler metric sits in the real Néron-Severi group $\left(H^{2}(X, \mathbf{Z}) \cap H^{1,1}(X, \mathbf{C})\right) \otimes \mathbf{R}$ as the base-point-free theorem holds for $\mathbf{R}$-divisors due to Shokurov.

\section{Base point freeness}

Let $X$ be an $n$-dimensional projective manifold and $L \rightarrow X$ a holomorphic line bundle over $X$. Let $N(L)$ be the semi-group defined by

$$
N(L)=\left\{m \in \mathbf{N} \mid H^{0}\left(X, L^{m}\right) \neq 0\right\} .
$$

Given any $m \in N(L)$, the linear system $\left|L^{m}\right|=\mathbf{P} H^{0}\left(X, L^{m}\right)$ induces a rational map

$$
\Phi_{m}: X \rightarrow \mathbf{C P}^{d_{m}}
$$

by any basis $\left\{\sigma_{m, 0}, \sigma_{m, 1}, \ldots, \sigma_{m, d_{m}}\right\}$ of $H^{0}\left(X, L^{m}\right)$ as

$$
\Phi_{m}(z)=\left[\sigma_{m, 0}, \sigma_{m, 1}, \ldots, \sigma_{m, d_{m}}\right](z)
$$

where $d_{m}+1=\operatorname{dim} H^{0}\left(X, L^{m}\right)$. Let $Y_{m}=\overline{\Phi_{m}(X)} \subset \mathbf{C P}^{d_{m}}$ be the closure of the image of $X$ by $\Phi_{m}$.

Definition 2.1. The Iitaka dimension of $L$ is defined to be

$$
\kappa(X, L)=\max _{m \in N(L)}\left\{\operatorname{dim} Y_{m}\right\}
$$

if $N(L) \neq \phi$, and $\kappa(X, L)=-\infty$ if $N(L)=\phi$.

Definition 2.2. Let $X$ be an projective manifold and $K_{X}$ the canonical line bundle over $X$. Then the Kodaira dimension $\operatorname{kod}(X)$ of $X$ is defined to be

$$
\operatorname{kod}(X)=\kappa\left(X, K_{X}\right)
$$


The Kodaira dimension is a birational invariant of projective varieties and the Kodaira dimension of a singular variety is equal to that of its smooth model.

Definition 2.3. Let $L \rightarrow X$ be a holomorphic line bundle over a projective manifold $X . L$ is called semi-ample if the linear system $\left|L^{m}\right|$ is base point free for some $m>0$.

For any $m \in \mathbf{N}$ such that $\left|L^{m}\right|$ is base point free, the linear system $\left|L^{m}\right|$ induces a holomorphic map $\Phi_{m}$

$$
\Phi_{m}: X \rightarrow \mathbf{C P}^{d_{m}}
$$

by any basis of $H^{0}\left(X, L^{m}\right)$. Let $Y_{m}=\Phi_{m}(X)$ and so

$$
\Phi_{m}: X \rightarrow Y_{m} \in \mathbf{C P}^{d_{m}} .
$$

The following theorem is well known (see [L, U]).

Theorem 2.1. Let $L \rightarrow X$ be a semi-ample line bundle over an algebraic manifold $X$. Then there is a projective fibration

$$
\pi: X \rightarrow Y
$$

such that for any sufficiently large integer $m$ with $L^{m}$ being globally generated,

$$
Y_{m}=Y \quad \text { and } \quad \Phi_{m}=\pi,
$$

where $Y$ is a normal projective variety.

Furthermore, there exists an ample line bundle $A$ on $Y$ such that $L^{m}=$ $\pi^{*} A$.

If $L$ is semi-ample, the graded $\operatorname{ring} R(X, L)=\oplus_{m \geq 0} H^{0}\left(X, L^{m}\right)$ is finitely generated and so $R(X, L)=\oplus_{m \geq 0} H^{0}\left(X, L^{m}\right)$ is the coordinate ring of $Y$.

Definition 2.4. Let $L \rightarrow X$ be a semi-ample line bundle over a projective manifold $X$. Then the algebraic fibration $\pi: X \rightarrow Y$ as in Theorem 2.1 is called the Iitaka fibration associated with $L$. It is completely determined by the linear system $\left|L^{m}\right|$ for sufficiently large $m$.

The following theorems are known as the rationality theorem and basepoint-free theorem in the minimal model program (see [KMM, KM]). 
Theorem 2.2. Let $X$ be a projective manifold such that $K_{X}$ is not nef. Let $H$ be an ample divisor and let

$$
\lambda=\max \left\{t \in \mathbf{R} \mid H+t K_{X} \text { is nef }\right\} .
$$

Then $\lambda \in \mathbf{Q}$.

Theorem 2.3. Let $X$ be a projective manifold. Let $D$ be a nef divisor such that $a D-K_{X}$ is nef and big for some $a>0$. Then $D$ is semi-ample.

We now will apply the base-point-free theorem to the Kähler-Ricci flow at the finite blow-up time. We consider the unnormalized Kähler-Ricci flow (1.1) with the initial Kähler class $H=\left[\omega_{0}\right] \in H^{2}(X, \mathbf{Z}) \cap H^{1,1}(X, \mathbf{C})$. The evolution of the Kähler class satisfies the following ordinary differential equation

$$
\frac{\partial}{\partial t}[\omega]=\left[K_{X}\right],\left.\quad[\omega]\right|_{t=0}=[H] .
$$

Therefore, $[\omega]=\left[H+t K_{X}\right]$ for $t \geq 0$.

Lemma 2.1. Let

$$
T=\sup \left\{t \geq 0 \mid H+t K_{X} \text { is nef }\right\} .
$$

Then $T \in \mathbf{Q}$ if $K_{X}$ is not nef and $T=\infty$ if $K_{X}$ is nef.

Proof. The lemma is an immediate corollary of the rationality Theorem 2.2 .

The following theorem is proved in [TZha] for the maximal existence of the Kähler-Ricci flow.

Proposition 2.1. The Kähler-Ricci flow (1.1) exists for $t \in[0, T)$.

Lemma 2.2. Let $L=H+T K_{X}$. Then $L$ is semi-ample.

Proof. Notice that $L-\epsilon K_{X}=H+(T-\epsilon) K_{X}$ is ample for sufficiently small $\epsilon>0$. The lemma follows from the base-point-free theorem 2.3. 


\section{The case when $K_{X}$ is not nef and $\kappa(L)=0$}

If $K_{X}$ is not nef, $T<\infty$ and the unnormalized Kähler-Ricci flow (1.1) does not have long-time existence and it must develop singularities at $t=T$. In particular, it is shown in [Z] that the scalar curvature must blow up at $t=T$. Let $L=H+T K_{X}$. Then either $\kappa(L)=0$ or $\kappa(L)>0$ as $L$ is semi-ample.

Proposition 3.1. If $\kappa(L)=0, X$ is Fano and $[H]$ is proportional to $c_{1}(X)$.

Proof. Since $L$ semi-ample and $\kappa(L)=0, L^{m}$ is trivial for some $m$ and $[L]=$ 0 . Hence

$$
T c_{1}(X)=-T\left[K_{X}\right]=[H]>0 \text {. }
$$

The following theorem is proved by Perelman [P2] (see [SeT]).

Theorem 3.1. Let $X$ be a Fano manifold of complex dimension $n \geq 2$. The unnormalized Kähler-Ricci flow becomes extinct in finite time if the initial Kähler class is proportional to the first Chern class $c_{1}(X)$.

More precisely, Perelman shows that if $X$ is Fano and the initial Kähler metric lies in $c_{1}(X)$, the diameter of the evolving metrics is uniformly bounded above along the normalized Kähler-Ricci flow $\frac{\partial}{\partial t} g=-\operatorname{Ric}(g)+g$. The above theorem follows immediately after scaling the normalized flow back to the unnormalized flow.

\section{The case when $K_{X}$ is not nef and $\kappa(L)>0$}

Now we assume $k=\kappa(L)>0$. Since $L$ is semi-ample, $H^{0}\left(X, L^{m}\right)$ induces a holomorphic map for sufficiently large $m$

$$
\pi: X \rightarrow Y \subset \mathbf{C P}^{d_{m}}
$$

where $Y$ is a normal variety of $\operatorname{dim} Y=k$ and $d_{m}+1=\operatorname{dim} H^{0}\left(X, L^{m}\right)$.

Since $L=H+T K_{X}$ is semi-ample, there exists a smooth $(1,1)$-form $\chi \in\left[K_{X}\right]$ such that

$$
\omega_{T}=\omega_{0}+T \chi \geq 0
$$

is proportional to the pullback of the Fubini-Study metric $\omega_{\mathrm{FS}}$ on $\mathbf{C P}^{d_{m}}$ by $\pi$. There also exists a smooth volume form $\Omega$ on $X$ such that

$$
\sqrt{-1} \partial \bar{\partial} \log \Omega=\chi
$$


Let $\omega_{t}=\omega_{0}+t \chi$. Then

$$
\omega_{t}=\frac{t}{T} \omega_{T}+\frac{T-t}{T} \omega_{0} \geq \frac{T-t}{T} \omega_{0}>0
$$

and the following lemma holds immediately.

Lemma 4.1. For any $(t, z) \in[0, T) \times X$,

$$
\frac{\omega_{t}^{n}}{\omega_{0}^{n}} \geq\left(\frac{T-t}{T}\right)^{n} .
$$

We consider the following Monge-Ampère flow induced by the unnormalized Kähler-Ricci flow.

$$
\left\{\begin{array}{l}
\frac{\partial}{\partial t} \varphi=\log \frac{\left(\omega_{t}+\sqrt{-1} \partial \bar{\partial} \varphi\right)^{n}}{\Omega} \\
\left.\varphi\right|_{t=0}=0 .
\end{array}\right.
$$

Lemma 4.2. There exists $C>0$ such that for $(t, z) \in[0, T) \times X$,

$$
\varphi \leq C
$$

Proof. Let $\varphi_{\max }(t)=\max _{z \in X} \varphi(t, z)$. Then

$$
\frac{\partial}{\partial t} \varphi_{\max } \leq \log \frac{\omega_{t}^{n}}{\Omega} \leq \log \frac{\left(\omega_{0}+\omega_{T}\right)^{n}}{\Omega} \leq C .
$$

Hence

$$
\varphi(t, z) \leq C T
$$

Proposition 4.1. There exists $C>0$ such that for all $(t, z) \in[0, T) \times X$,

$$
\operatorname{tr}_{\omega}\left(\omega_{T}\right) \leq C
$$

Proof. This is a parabolic Schwarz lemma similar to the one given in [SoT1]. Suppose $\omega_{T}=c \pi^{*} \omega_{\mathrm{FS}}$ and $\omega_{\mathrm{FS}}=h_{\alpha \bar{\beta}} d x^{\alpha} \wedge d \bar{x}^{\beta}$.

Choose normal coordinate systems for $g=\omega(t, \cdot)$ on $X$ and $h$ on $\mathbf{C P}^{d_{m}}$, respectively. Let $u=\operatorname{tr}_{g}(h)=g^{i \bar{j}} \pi_{i}^{\alpha} \pi_{\bar{j}}^{\bar{\beta}} h_{\alpha \bar{\beta}}$ and we will calculate the evolution of $u$. $u$ is nonnegative as $\pi$ is holomorphic. Standard calculation 
shows that

$$
\begin{aligned}
\Delta u & =g^{k \bar{l}} \partial_{k} \partial_{\bar{l}}\left(g^{i \bar{j}} \pi_{i}^{\alpha} \pi_{\bar{j}}^{\bar{\beta}} h_{\alpha \bar{\beta}}\right) \\
& =g^{i \bar{l}} g^{k \bar{j}} R_{k \bar{l}} \pi_{i}^{\alpha} \pi_{\bar{j}}^{\bar{\beta}} h_{\alpha \bar{\beta}}+g^{i \bar{j}} g^{k \bar{l}} \pi_{i, k}^{\alpha} \pi_{\bar{j}, \bar{l}}^{\bar{\beta}} h_{\alpha \bar{\beta}}-g^{i \bar{j}} g^{k \bar{l}} S_{\alpha \bar{\beta} \gamma \bar{\delta}} \pi_{i}^{\alpha} \pi_{\bar{j}}^{\bar{\beta}} \pi_{k}^{\gamma} \pi_{\bar{l}}^{\bar{\delta}},
\end{aligned}
$$

where $S_{\alpha \bar{\beta} \gamma \bar{\delta}}$ is the curvature tensor of $h_{\alpha \bar{\beta}}$. By the definition of $u$ we have

$$
\Delta u \geq g^{i \bar{l}} g^{k \bar{j}} R_{k \bar{l}} \pi_{i}^{\alpha} \pi_{\bar{j}}^{\bar{\beta}} h_{\alpha \bar{\beta}}-K u^{2}+g^{i \bar{j}} g^{k \bar{l}} \pi_{i, k}^{\alpha} \pi_{\bar{j}, \bar{l}}^{\bar{\beta}} h_{\alpha \bar{\beta}}
$$

for some fixed constant $K>0$. Now

$$
\begin{aligned}
\frac{\partial u}{\partial t} & =-g^{i \bar{l}} g^{k \bar{j}} \frac{\partial g_{k \bar{l}}}{\partial t} \pi_{i}^{\alpha} \pi_{\bar{j}} h_{\alpha \bar{\beta}} \\
& =g^{i \bar{l}} g^{k \bar{j}} R_{k \bar{l}} \pi_{i}^{\alpha} \pi_{\bar{j}}^{\bar{\beta}} h_{\alpha \bar{\beta}},
\end{aligned}
$$

therefore

$$
\begin{aligned}
\left(\frac{\partial}{\partial t}-\Delta\right) \log u & =\frac{1}{u}\left(\frac{\partial}{\partial t}-\Delta\right) u-\frac{|\nabla u|^{2}}{u^{2}} \\
& \leq K u-\frac{1}{u^{2}}\left(u g^{i \bar{j}} g^{k \bar{l}} \pi_{i, k}^{\alpha} \pi_{\bar{j}, \bar{\beta}}^{\bar{\beta}} h_{\alpha \bar{\beta}}-|\nabla u|^{2}\right) \\
& \leq K u,
\end{aligned}
$$

where the last inequality follows from $u g^{i \bar{j}} g^{k \bar{l}} \pi_{i, k}^{\alpha} \pi_{\bar{j}, \bar{l}}^{\bar{\beta}} h_{\alpha \bar{\beta}}-|\nabla u|^{2} \geq 0$ (see p. 627 [SoT1]).

Consider $H=\log u-2 A \varphi$. If $A$ is chosen to be sufficiently large,

$$
\begin{aligned}
& \left(\frac{\partial}{\partial t}-\Delta\right) H \\
& \quad \leq-\operatorname{tr}_{\omega}\left(2 A \omega_{t}-K \omega_{T}\right)-2 A \log \frac{\omega^{n}}{\Omega}+2 A n \\
& \quad \leq-\operatorname{tr}_{\omega}\left(A \omega_{t}\right)+2 A \log \frac{\omega_{t}^{n}}{\omega^{n}}+2 A \log \frac{\Omega}{\omega_{t}^{n}}+2 A n \\
& \quad \leq-C A n \log (T-t)+C A
\end{aligned}
$$

$\left.H\right|_{t=0}=\log \operatorname{tr}_{\omega_{0}}\left(\omega_{T}\right)$ is bounded from above. By the maximum principle,

$$
H \leq-C \int_{0}^{T} \log (T-t) d t+C \leq C^{\prime} .
$$

The proposition is proved as $\varphi$ is uniformly bounded from above. 
Corollary 4.1. There exists $C>0$ such that for $(t, z) \in[0, T) \times X$

$$
\omega_{t} \geq C \pi^{*} \omega_{\mathrm{FS}}
$$

Theorem 4.1. Let $g(t)$ be the Kähler metric associated with $\omega(t, \cdot)$. Then there exists $C>0$, such that for $t \in[0, T)$,

$$
\operatorname{diam}(X, g(t)) \geq C \text {. }
$$

Proof. Since $Y$ is normal, the singular set of $Y$ is an analytic subvariety of $Y$ of codimension greater than one. Let $x_{0}$ be a point in the nonsingular part $Y_{\text {reg }}$ of $Y$. Then there exists a geodesic ball $B_{2 r}\left(x_{0}, g_{\mathrm{FS}}\right) \subset Y_{\text {reg }}$ of radius $2 r>0$ centered at $x_{0}$ with respect to the Fubini-Study metric $g_{\mathrm{FS}}$ restricted on $Y$. There exist $x_{1}$ and $x_{2} \in B_{r}\left(x_{0}, g_{\mathrm{FS}}\right)$ such

$$
d_{Y, g_{\mathrm{FS}}}\left(x_{1}, x_{2}\right) \geq r
$$

where $d_{Y, g_{\mathrm{FS}}}\left(x_{1}, x_{2}\right)$ is the distance between $x_{1}$ and $x_{2}$ on $Y$ with respect to $g_{\mathrm{FS}}$.

Choose $z_{1} \in \pi^{-1}\left(x_{1}\right)$ and $z_{2} \in \pi^{-1}\left(x_{2}\right)$. Then for $i=1,2$, let

$$
d_{\pi^{-1}\left(B_{2 r}\left(x_{0}, g_{\mathrm{FS}}\right)\right), g(t)}\left(z_{i}, \pi^{-1} \partial\left(B_{2 r}\left(x_{0}, g_{\mathrm{FS}}\right)\right)\right)
$$

be the distance from $z_{i}$ to $\pi^{-1} \partial\left(B_{2 r}\left(x_{0}, g_{\mathrm{FS}}\right)\right)$ in $\pi^{-1}\left(B_{2 r}\left(x_{0}, g_{\mathrm{FS}}\right)\right)$ with respect to $g(t)$ and $d_{Y, g_{\mathrm{FS}}}\left(x_{i}, \partial B_{2 r}\left(x_{0}, g_{\mathrm{FS}}\right)\right)$ be the distance from $x_{i}$ to $\partial B_{2 r}\left(x_{0}, g_{\mathrm{FS}}\right)$ on $Y$ with respect to $g_{\mathrm{FS}}$. Then

$$
\begin{aligned}
& d_{\pi^{-1}\left(B_{2 r}\left(x_{0}, g_{\mathrm{FS}}\right)\right), g(t)}\left(z_{i}, \pi^{-1} \partial\left(B_{2 r}\left(x_{0}, g_{\mathrm{FS}}\right)\right)\right) \\
& \quad \geq C d_{Y, g_{\mathrm{FS}}}\left(x_{i}, \partial B_{2 r}\left(x_{0}, g_{\mathrm{FS}}\right)\right) \geq C r,
\end{aligned}
$$

and so

$$
d_{X, g(t)}\left(z_{1}, z_{2}\right) \geq C d_{Y, g_{\mathrm{FS}}}\left(x_{1}, x_{2}\right) \geq C r .
$$

\section{The case when $K_{X}$ is nef}

When $K_{X}$ is nef, it follows from Proposition 2.1 that the unnormalized Kähler-Ricci flow (1.1) has long-time existence. 
Proposition 5.1. Let $X$ be $n$-dimensional projective manifold of $\operatorname{kod}(X)=0$. If $K_{X}$ is semi-ample, then there exists $C>0$ depending on $g_{0}$ such that along the Kähler-Ricci flow (1.1)

$$
\operatorname{diam}(X, g(t)) \geq C
$$

Proof. If $\operatorname{kod}(X)=0$ and $K_{X}$ is semi-ample, $c_{1}(X)=0$. The proposition is a result of $\mathrm{Cao}[\mathrm{Cao}, \mathrm{Y} 1]$.

Proposition 5.2. Let $X$ be $n$-dimensional projective manifold of $\operatorname{kod}(X)>0$. If $K_{X}$ is semi-ample, then there exists $C>0$ depending on $g_{0}$ such that along the Kähler-Ricci flow (1.1)

$$
\operatorname{diam}(X, g(t)) \geq C \sqrt{t}
$$

Proof. We consider the following normalized Kähler-Ricci flow when $K_{X}$ is nef.

$$
\left\{\begin{array}{l}
\frac{\partial}{\partial s} \tilde{g}=-\operatorname{Ric}(\tilde{g})-\tilde{g}, \\
\left.\tilde{g}\right|_{s=0}=g_{0} .
\end{array}\right.
$$

The relation between the solution $g(t)$ of the unnormalized Kähler-Ricci flow (1.1) and the solution $\tilde{g}(s)$ of the normalized flow (5.3) is given by

$$
\begin{aligned}
t & =e^{s}-1, \\
g(t)=e^{s} \tilde{g}(s) & =(t+1) \tilde{g}(\log (t+1)) .
\end{aligned}
$$

If $X$ is of general type, there exists a birational holomorphic map $f$ : $X \rightarrow X_{\text {can }}$ from $X$ to its canonical model $X_{\text {can }}$ as $R\left(X, K_{X}\right)$ is finitely generated. Let $E$ be the set of all the points on $X$ such that $f$ is not isomorphic. Then $E$ is a subvariety of $X$ and it is proved in [Ts, TZha] that $g(t)$ converges in $C^{\infty}(X \backslash E)$ as $t \rightarrow \infty$ along the normalized flow (5.3). Since the limiting singular Kähler-Einstein metric is smooth and nondegenerate on $X \backslash E, \operatorname{diam}(X, \tilde{g}(t))$ is then uniformly bounded below from 0 .

If $0<k=\operatorname{kod}(X)<n$, then there exists a unique holomorphic map $f$ : $X \rightarrow X_{\text {can }} \in \mathbf{C P}^{N}$, where $X_{\text {can }}$ is the canonical model of $X$ of 
$\operatorname{dim} X_{\text {can }}=k$. Let

$$
X_{\text {can }}^{\circ}=\left\{x \in X_{\text {can }} \mid x \text { is nonsingular and } f^{-1}(x) \text { is nonsingular }\right\} .
$$

Let $h$ be the pullback of the Fubini-Study metric on $X_{\text {can }}$. Then it is proved in [SoT1, SoT2] by the parabolic Schwarz lemma, that for any $K \subset \subset X_{\text {can }}^{\circ}$, there exists $C_{K}>0$ such that on $[0, \infty) \times K$,

$$
\tilde{g}(t) \geq C_{K} f^{*} h
$$

along the normalized Kähler-Ricci flow (5.3). Therefore, $\operatorname{diam}(X, \tilde{g}(t))$ is uniformly bounded from below from 0 .

Combining the above estimates, for any initial metric $g_{0}$, there exists $C>0$ such that along the normalized Kähler-Ricci flow (5.3),

$$
\operatorname{diam}(X, \tilde{g}(s)) \geq C
$$

The proposition is proved as $g(t)=(t+1) \tilde{g}(\log (t+1))$.

Now we are able to prove the main theorems.

Proof of Theorem 1.2. If $K_{X}$ is not nef and $K_{X}^{-1}$ is not ample, $L=H+$ $T K_{X}$ has positive Iitaka dimension at the singular time $t=T$. If $K_{X}^{-1}$ is ample and $\left[\omega_{0}\right]$ is not proportional to $K_{X}^{-1}, K_{X}$ is not nef and $L=H+T K_{X}$ has positive Iitaka dimension at the singular time $t=T$ by Proposition 3.1. Then the proposition follows by combining Theorem 4.1, Propositions 5.1 and 5.2.

Proof of Theorem 1.1. The Kähler-Ricci flow (1.1) has long-time existence if $K_{X}$ is nef. Theorem 1.1 follows easily from Theorems 1.2 and 3.1.

\section{Acknowledgements}

The author is grateful to Professor D.H. Phong for his advice, encouragement and support. The author would like to thank: Professor G. Tian for bringing his article [T2] to his attention as well as his useful suggestion; V. Tosatti for a number of very enlightening discussions; Professor J. Sturm, G. Szekelyhidi, B. Weinkove and Y. Yuan for helpful suggestions. Research supported in part by National Science Foundation grant DMS-06-04805. 


\section{References}

[A] T. Aubin, Équations du type Monge-Ampère sur les variétés kählériennes compactes, Bull. Sci. Math. (2) 102(1) (1978), 63-95.

[Cao] H.-D. Cao, Deformation of Kähler metrics to Kähler-Einstein metrics on compact Kähler manifolds, Invent. Math. 81(2) (1985), 359-372.

[CW] X.X. Chen and B. Wang, Space of Ricci flows I, Comm. Pure Appl. Math. 65(10) (2012), 1399-1457

[Ch] B. Chow, The Ricci flow on the 2-sphere, J. Differential Geom. 33 (1991), 325-334.

[CS] T. Collins and G. Szekelyhidi, The twisted Kähler-Ricci flow, 2012, arXiv: 1207.5441

[D] S.K. Donaldson, Scalar curvature and stability of toric varieties, J. Differential Geom. 62(2) (2002), 289-349.

[H] R.S. Hamilton, The Ricci flow on surfaces, Contemp. Math. 71 (1988), 237-261.

[IK] T. Ilmanen and D. Knopf, A lower bound for the diameter of solutions to the Ricci flow with nonzero $H^{1}\left(M^{n} ; R\right)$, Math. Res. Lett. 10(2) (2003), 161-168.

[L] J. Lazarsfeld, Positivity in algebraic geometry. I. Classical setting: line bundles and linear series, in 'A series of modern surveys in mathematics', 48, Springer-Verlag, Berlin, 2004, xviii+387 pp.

[KMM] Y. Kawamata, K. Matsuda and K. Matsuki, Introduction to the minimal model problem, Algebraic geometry, Sendai, 1985, Adv. Stud. Pure Math., 10, North-Holland, Amsterdam, 1987, 283-360.

[KM] S. Kollár and S. Mori, Birational geometry of algebraic varieties, in 'Cambridge tracts in mathematics', 134, Cambridge University Press, Cambridge, 1998.

[P1] G. Perelman, The entropy formula for the Ricci flow and its geometric applications, 2002, arXiv:math.DG/0211159.

[P2] _ unpublished work on the Kähler-Ricci flow.

[PS1] D.H. Phong and J. Sturm On the Kähler-Ricci flow on complex surfaces, Pure Appl. Math. Q. 1(2) (2005), Part 1, 405-413 
[PS2] _ On stability and the convergence of the Kähler-Ricci flow, J. Differential Geom. 72(1) (2006), 149-168

[PSSW1] D.H. Phong, J. Song, J. Sturm and B. Weinkove, The KählerRicci flow and the $\bar{\partial}$ operator on vector fields, J. Differential Geom. 81(3) (2009), 631-647

[PSSW2] _ The modified Kähler-Ricci flow and solitons, Comment. Math. Helv. 86(1) (2011), 91-112

[SeT] N. Sesum and G. Tian Bounding scalar curvature and diameter along the Kähler Ricci flow (after Perelman), J. Inst. Math. Jussieu 7(3) (2008), 575-587

[SoT1] J. Song and G. Tian, The Kähler-Ricci flow on surfaces of positive Kodaira dimension, Invent. Math. 170(3) (2007), 609-653

[SoT2] _ Canonical measures and Kähler-Ricci flow, J. Amer. Math. Soc. 25(2) (2012), 303-353

[SoT3] , The Kähler-Ricci flow through singularities, 2009, arXiv:0909.4898

[SW] J. Song and B. Weinkove, The Kähler-Ricci flow on Hirzebruch surfaces, J. Reine Angew. Math. 659 (2011), 141-168

[Sz] G. Szekelyhidi, The Kähler-Ricci flow and K-stability, Amer. J. Math. 132(4) (2010), 1077-1090

[T1] G. Tian, Kähler-Einstein metrics with positive scalar curvature, Invent. Math. 130(1) (1997), 1-37

[T2] _ New results and problems on Kähler-Ricci flow, in 'Geometrie differentielle, physique mathematique, mathematiques et societe', II. Asterisque No. 322 (2008), 71-92

[TZha] G. Tian and Z. Zhang, On the Kähler-Ricci flow on projective manifolds of general type, Chinese Ann. Math. Ser. B 27(2) (2006), 179-192

[TZhu] G. Tian and X. Zhu, Convergence of Kähler-Ricci flow, J. Amer. Math. Soc. 20(3) (2007), 675-699

[To] V. Tosatti, Kähler-Ricci flow on stable Fano manifolds, J. Reine Angew. Math. 640 (2010), 67-84

[Ts] H. Tsuji, Existence and degeneration of Kähler-Einstein metrics on minimal algebraic varieties of general type, Math. Ann. 281 (1988), $123-133$ 
[U] K. Ueno, Classification theory of algebraic varieties and compact complex spaces, notes written in collaboration with P. Cherenack, Lecture Notes in Mathematics, Vol. 439, Springer-Verlag, Berlin-New York, 1975, xix +278 pp.

[Y1] S.-T. Yau, On the Ricci curvature of a compact Kähler manifold and the complex Monge-Ampère equation, I, Comm. Pure Appl. Math. 31 (1978), 339-411

[Y2] Open problems in geometry, Proc. Symposia Pure Math. 54 (1993), 1-28 (problem 65)

[Z] Z. Zhang, Scalar curvature behavior for finite time singularity of Kähler-Ricci flow, Michigan Math. J. 59(2) (2010), 419-433

Department of Mathematics

RutGers UNIVERSITY

PiscataWAY, NJ 08854

E-mail address: jiansong@math.rutgers.edu

ReCeived November 5, 2013 
